\title{
Orbital-selective Mott transitions in the anisotropic two-band Hubbard model at finite temperatures
}

\author{
C. Knecht, N. Blümer * and P. G. J. van Dongen \\ Institute of Physics, Johannes Gutenberg University, 55099 Mainz, Germany
}

(Dated: August 15, 2018)

\begin{abstract}
The anisotropic degenerate two-orbital Hubbard model is studied within dynamical mean-field theory at low temperatures. High-precision calculations on the basis of a refined quantum Monte Carlo (QMC) method reveal that two distinct orbital-selective Mott transitions occur for a bandwidth ratio of 2 even in the absence of spin-flip contributions to the Hund exchange. The second transition - not seen in earlier studies using QMC, iterative perturbation theory, and exact diagonalization - is clearly exposed in a low-frequency analysis of the self-energy and in local spectra.

PACS numbers: 71.30.+h, 71.10.Fd, 71.27.+a
\end{abstract}

The Mott-Hubbard metal-insulator transition - a nonperturbative correlation phenomenon - has been a subject of fundamental interest in solid state theory for decades,$\frac{1}{m}$ Recently, this field became even more exciting by the discovery ${ }^{2.3}$ of a two-step metal-insulator transition in the effective 3-band system $\mathrm{Ca}_{2-x} \mathrm{Sr}_{x} \mathrm{RuO}_{4}$, for which the name orbital-selective Mott-transition (OSMT) was coined $\stackrel{4}{*}$ The $\mathrm{Ca}_{2-x} \mathrm{Sr}_{x} \mathrm{RuO}_{4}$ system was investigated theoretically in detail by Anisimov et $a l^{\underline{4}}$ within the local density approximation (LDA and $\mathrm{LDA}+\mathrm{U})$ and within dynamical mean-field theory ${ }^{5}$ (DMFT) solved using the non-crossing approximation (NCA). The underlying assumption of a correlation (rather than lattice-distortion) induced OSMT found support in further band structure calculations ${ }^{6.7}$ and strong-coupling expansions for the localized electrons in the orbital-selective Mott phase 8

Microscopic studies of the OSMT usually consider the 2-band Hubbard model $H=H_{1}+H_{2}$, where

$$
\begin{aligned}
H_{1}= & -\sum_{\langle i j\rangle m \sigma} t_{m} c_{i m \sigma}^{\dagger} c_{j m \sigma}+U \sum_{i m} n_{i m \uparrow} n_{i m \downarrow} \\
& +\sum_{i \sigma \sigma^{\prime}}\left(U^{\prime}-\delta_{\sigma \sigma^{\prime}} J_{z}\right) n_{i 1 \sigma} n_{i 2 \sigma^{\prime}}
\end{aligned}
$$

includes hopping between nearest-neighbor sites $i, j$ with amplitude $t_{m}$ for orbital $m \in\{1,2\}$, intra- and interorbital Coulomb repulsion parametrized by $U$ and $U^{\prime}$, respectively, and Ising-type Hund's exchange coupling; $n_{i m \sigma}=c_{i m \sigma}^{\dagger} c_{i m \sigma}$ for spin $\sigma \in\{\uparrow, \downarrow\}$. In addition,

$$
H_{2}=\frac{1}{2} J_{\perp} \sum_{i m \sigma} c_{i m \sigma}^{\dagger}\left(c_{i \bar{m} \bar{\sigma}}^{\dagger} c_{i m \bar{\sigma}}+c_{i m \bar{\sigma}}^{\dagger} c_{i \bar{m} \bar{\sigma}}\right) c_{i \bar{m} \sigma}
$$

contains spin-flip and pair-hopping terms (with $\overline{1} \equiv 2$, $\bar{\uparrow} \equiv \downarrow$ etc.). In cubic lattices, the Hamiltonian is invariant under spin rotation, $J_{z}=J_{\perp} \equiv J$; furthermore $U^{\prime}=$ $U-2 J$. In the following, we refer to $H_{1}+H_{2}$ in this spin-isotropic case as the $J$-model and to the simplified Hamiltonian $H_{1}$ as the $J_{z}$-model.

Liebsch $9.10,11$ questioned the OSMT scenario for $\mathrm{Ca}_{2-x} \mathrm{Sr}_{x} \mathrm{RuO}_{4}$ on the basis of finite-temperature quantum Monte Carlo (QMC) calculations (within DMFT) for the $J_{z}$-model using $J_{z}=U / 4, U^{\prime}=U / 2$, and semielliptic "Bethe" densities of states with a bandwidth ra- tio $W_{2} / W_{1}=2$. Additional studies using iterative perturbation theory (IPT) $)^{11}$ seemed $^{12}$ to confirm his conclusion of a single Mott transition of both bands at the same critical $U$-value. Meanwhile, Koga et al. found an OSMT using exact diagonalization (ED), applied to the full $J$-model, 13 but not for the $J_{z}$-model ${ }^{14}$ Consequently, the OSMT scenario was attributed to spin-flip and pairhopping processes.

Very recently, four preprints appeared, 15.16.17.18 in which the OSMT was investigated in detail within the DMFT framework. Ref. 15 applied the Gutzwiller variational approach and ED to the $J$-model at temperature $T=0$ and confirmed the existence of an OSMT, provided that the ratio $W_{2} / W_{1}$ of the two band widths is sufficiently small. Interestingly, Ref. 15 also suggested the existence of small spectral weight near the Fermi level of the narrow-band subsystem in the orbital-selective Mott phase. Similar results were obtained by de' Medici et $a l ., 16$ who used slave-spin mean field theory (which is closely related to the Gutzwiller method). Arita and Held $^{17}$ used the projective QMC method to investigate the $J$-model at $T=0$ and demonstrated a first OSMT for $J=U / 4$ and $U=2.6$ (in units of half the narrow-band width). Finally, Koga et al ${ }^{14}$ used QMC to characterize the OSMT for the $J$-model at finite $T$ on the basis of spin, charge and orbital susceptibilities as well as spectral functions; they further showed that additional hybridization between the bands smears out the OSMT at $T=0$. Remarkably, the problem originally investigated by Liebsch, $, 9,10,11$ i. e., the $J_{z}$-model with $U^{\prime}=U-2 J_{z}$ was addressed only in Ref. 16, where de' Medici et al. found an OSMT at $T=0$ within slave-spin mean-field theory, in contradiction to Liebsch's and Koga's earlier results. Since the slave-spin method is essentially uncontrolled, the existence of an OSMT in the $J_{z}$-model therefore remains unclear.

The goal of this work is to clarify this issue and to establish whether $H_{1}$ can be regarded as a minimal model for the OSMT. In the following, we will sketch our highprecision DMFT algorithm, which supplements QMC by a high-frequency expansion of the self-energy, before we discuss relevant observables and data-analysis techniques and present numerical results. 
Quantum Monte Carlo scheme - Within the DMFT, the lattice problem is mapped onto a single-impurity Anderson model with general hybridization, which has to be determined self-consistently $\underline{\underline{5}}$ DMFT algorithms are characterized by the numerical method employed in the solution of the impurity problem and by the iterative procedure used in order to establish self-consistency. In this work, the imaginary-time Green function $G(\tau)$ of the multi-band impurity is obtained in discretized form from Hirsch-Fye QMC simulations. Since the lattice Dyson equation is most easily formulated in the frequency domain, Fourier transformations (FTs) are needed twice per DMFT self-consistency cycle. Since naive FTs would violate the analytic properties of Green functions and self-energies, all DMFT-QMC codes use either a special transformation ${ }^{19}$ or interpolate the discrete QMC data by cubic splines $\frac{5.20}{20}$ Recently, it was realized 21.22 that natural boundary conditions are not well suited for modeling imaginary-time Green functions and that misfits can be reduced by allowing for non-zero second derivatives of $G$ at the boundaries $\tau \rightarrow 0, \tau \rightarrow \beta$ 늘 However, this improvement cannot eliminate misfits arising from higher order derivatives of $G$, which may be large at $0, \beta$ and are neglected in cubic splines.

Our DMFT-QMC method further improves on this situation by applying the FTs to a difference Green function $\Delta G \equiv G_{\mathrm{QMC}}-G_{\mathrm{HFE}}$, where the model Green function $G_{\mathrm{HFE}}$ is determined from a high-frequency expansion in such a way, that the second derivatives of $\Delta G(\tau)$ vanish and the higher derivatives are reduced at the boundaries. Consequently, natural cubic splines are appropriate for $\Delta G$. The full FT to Matsubara frequencies is then obtained as $G\left(i \omega_{n}\right)=\Delta G\left(i \omega_{n}\right)+G_{\mathrm{HFE}}\left(i \omega_{n}\right)$, where the last term is computed directly via the Dyson equation from the model self-energy $\Sigma_{\mathrm{HFE}}\left(i \omega_{n}\right)$. The latter is constructed on the basis of the asymptotic expansion ${ }^{21.23}$ $\Sigma_{\gamma}(\omega)=\sum_{\beta \neq \gamma} U_{\beta \gamma}\left\langle n_{\beta}\right\rangle+\Sigma_{\gamma}^{(2)}(\omega)+\mathcal{O}\left(\frac{1}{\omega^{2}}\right)$ with

$$
\Sigma_{\gamma}^{(2)}(\omega)=\frac{1}{\omega} \sum_{\alpha, \beta \neq \gamma} U_{\alpha \gamma} U_{\beta \gamma}\left(\left\langle n_{\alpha} n_{\beta}\right\rangle-\left\langle n_{\alpha}\right\rangle\left\langle n_{\beta}\right\rangle\right) .
$$

Here $\alpha, \beta$ and $\gamma$ are multi-indices, combining spin and orbital degrees of freedom: $\alpha \equiv(m, \sigma)$; the interaction matrix is defined by $U_{m \sigma, m \bar{\sigma}}=U, U_{m \sigma, \bar{m} \sigma^{\prime}}=U^{\prime}-J_{z} \delta_{\sigma \sigma^{\prime}}$. For the one-band model, where ${ }^{24} \Sigma_{\sigma}^{(2)}(\omega)=\left[U^{2}\left\langle n_{\bar{\sigma}}\right\rangle(1-\right.$ $\left.\left.\left\langle n_{\bar{\sigma}}\right\rangle\right)\right] / \omega$, the exceptional accuracy of our DMFT-QMC method has already been established in comparisons to semi-analytic extrapolated perturbation theory for the insulating phase ${ }^{25}$ Since the algorithm reduces the discretization error to the inevitable Trotter error, reliable QMC-data can be obtained already from a rather coarse discretization; the following results are based on QMC for $\Delta \tau=0.4$ unless indicated otherwise. The DMFT cycle was typically iterated 20 times with $10^{6}$ sweeps after convergency, using 1000 Matsubara frequencies. Full details of our algorithm will be presented elsewhere $\underline{26}$

Results - In the following, we present QMC results for the $J_{z}$-model with $J_{z}=U / 4, U^{\prime}=U / 2$ and semi-elliptic densities of states with full bandwidths $W_{1}=2, W_{2}=4$,

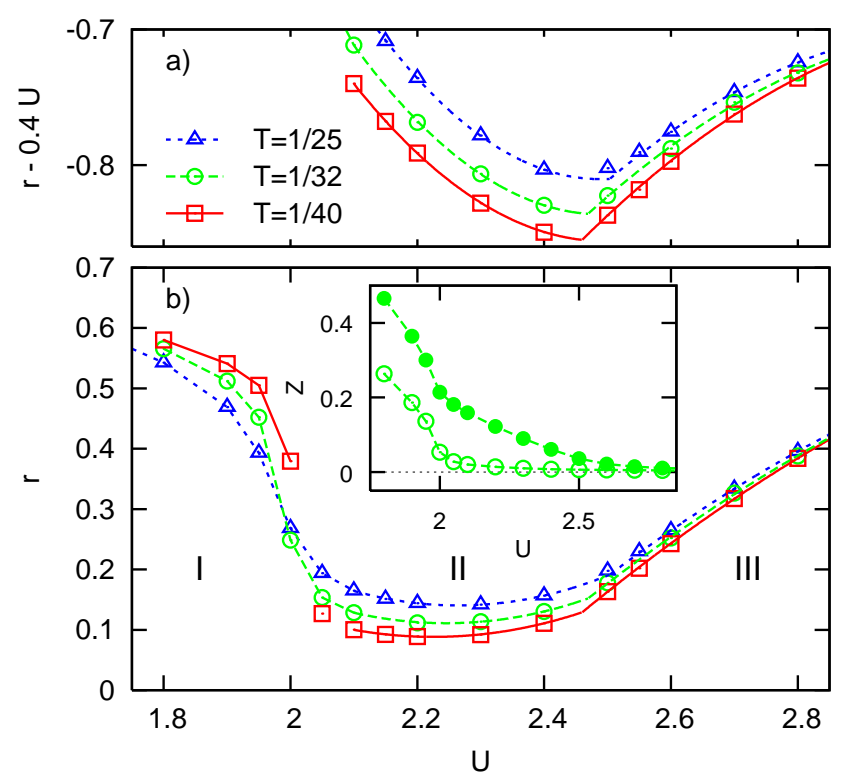

FIG. 1: (Color online) b) Ratio $r=Z_{\text {narrow }} / Z_{\text {wide }}$ of the discrete QMC estimates of the quasiparticle weights of both bands (shown in the inset) versus interaction $U$ for various temperatures $T$; lines for $U>2.1$ represent piecewise quadratic fits. a) Same data with linear offset.

for the "narrow" and "wide" band, respectively.

A traditional criterion for metal-insulator transitions is the quasiparticle weight $Z=\left[1-d \operatorname{Re} \Sigma /\left.d \omega\right|_{\omega=0}\right]^{-1}$ [for self-energy $\Sigma(\omega)$ ] which in the context of QMC simulations is estimated in a secant approximation as $Z \approx[1-\operatorname{Im} \Sigma(i \pi T) /(\pi T)]^{-1}$. Evidently both definitions recover the limit $Z \rightarrow 1$ in the absence of interactions (when $\Sigma \equiv 0$ ); however, in the insulating regime the discrete approximation necessarily remains finite, whereas the true $Z$ vanishes exactly. As a consequence, metalinsulator transitions are expected to appear washed-out at finite temperatures. This is seen in the inset of Fig. 10 for $T=1 / 32$ : $Z$ drops only by about $60 \%$ when the narrow band becomes insulating at $U \approx 2.0$. A second transition is not visible on this scale. The ratio $r \equiv Z_{\text {narrow }} / Z_{\text {wide }}$ shown in the main panel of Fig. पp is more illuminating since we can clearly distinguish three regions: in region I $(U \lesssim 2.0), r$ is of order unity with a sharp decrease near the boundary; in region II $(2.0 \lesssim U \lesssim 2.5), r$ is nearly constant and of order 0.1 , and in region III $(U \gtrsim 2.5), r$ increases with nearly constant slope (until it approaches a finite limit). In order to analyze the boundary between II and III, we have performed piecewise quadratic fits to the QMC data for both regions separately (lines for $U>2.1$ in Fig. 1 ). The kinks at the boundary, barely visible on this scale, but resolved after subtracting a linear offset in Fig. [1, indicate a second transition at least for $T \leq 1 / 32$ (for which all QMC data fall on the fit curves).

The intrinsic ambiguities associated with the discrete estimate for $Z$ can be overcome in a full low-frequency analysis of the self-energy as shown in Fig. 2 Here, the data points represent QMC estimates of the product 


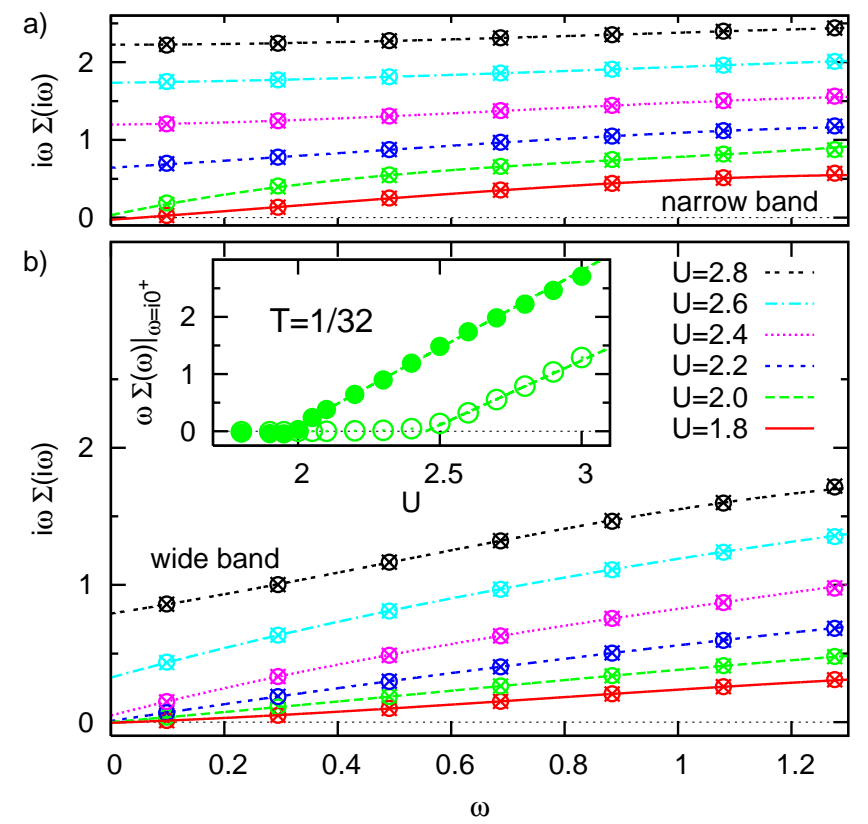

FIG. 2: (Color online) Low-frequency analysis of the selfenergy for $T=1 / 32$ : QMC data (crosses: for $\Delta \tau=0.40$, circles: for $\Delta \tau=0.32$ ) for the product of frequency $\omega$ and self-energy $\Sigma$ at the Matsubara frequencies is extrapolated by cubic polynomials in $\omega$ for the narrow/wide band (upper/lower panel). Inset: extrapolated value for $\omega \rightarrow i 0^{+}$.

$\omega \Sigma(\omega)$ at the Matsubara frequencies $i \omega_{n}$; these products are real-valued since the self-energy is purely imaginary on the imaginary axis due to particle-hole symmetry. The lines, given by cubic polynomials in $\omega$, are expected to extrapolate to 0 at least linearly within a metallic phase (where $\Sigma$ is regular) and to a finite value within an insulating phase. By this criterion, the narrow band (cf. Fig. 2 a) becomes insulating for $U \gtrsim 2.0$ while the wide band (cf. Fig. 2b) remains metallic up to $U=2.4$. Note that the QMC results obtained from discretizations $\Delta \tau=0.4$ (crosses) and $\Delta \tau=0.32$ (circles) agree on the scale of the figure. The extrapolated product $\left.\omega \Sigma(\omega)\right|_{\omega=i 0^{+}}$, a measure of the singularity in the self-energy and roughly proportional to the expected gap, is shown at better resolution as a function of $U$ in the inset of Fig. 20. We clearly see the presence of two distinct transitions for the narrow and wide band at $U=U_{c 1} \approx 2.0$ and $U=U_{c 2} \approx 2.5$, respectively. Corresponding results for higher and lower temperatures $(T=1 / 25$ and $T=1 / 40$, not shown) are barely distinguishable from Fig. 2 except for $U \approx U_{c 1}$.

The spectral function $N(\omega)=-\operatorname{Im} G(\omega) / \pi$, obtained from QMC by analytic continuation using the maximumentropy method (MEM), is depicted in Fig. 3] Evidently, $N(\omega=0)$ is accurately pinned at its noninteracting value up to $U=1.8$ for the wide band (lower panel) at $T=1 / 40$ and nearly pinned for the narrow band (upper panel). The narrow band develops a gap for $U>2.05$, where the wide band is still clearly metallic with a nearflat DOS at low frequencies. Only for $U=2.6$ a gap appears also for the wide band; note that the corresponding

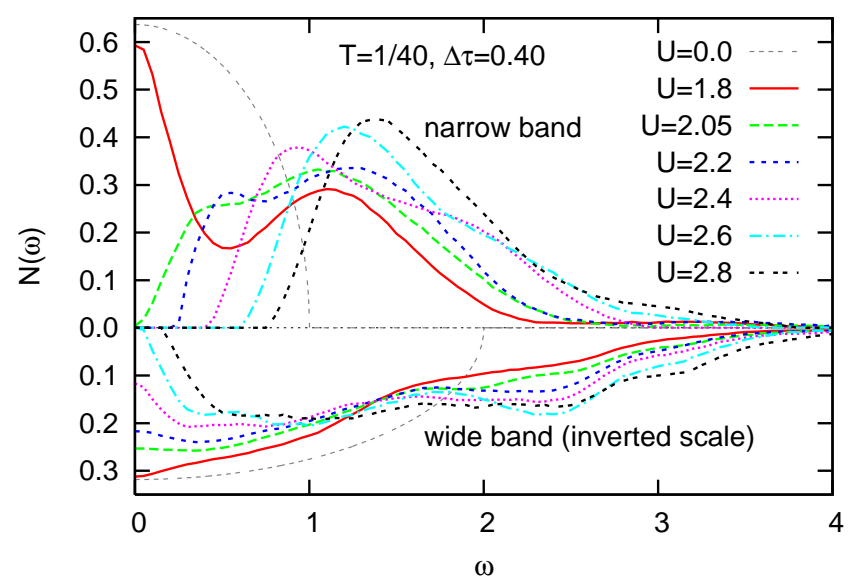

FIG. 3: (Color online) Spectral function of narrow/wide band (upper/lower panel) from QMC + MEM for $T=1 / 40$.

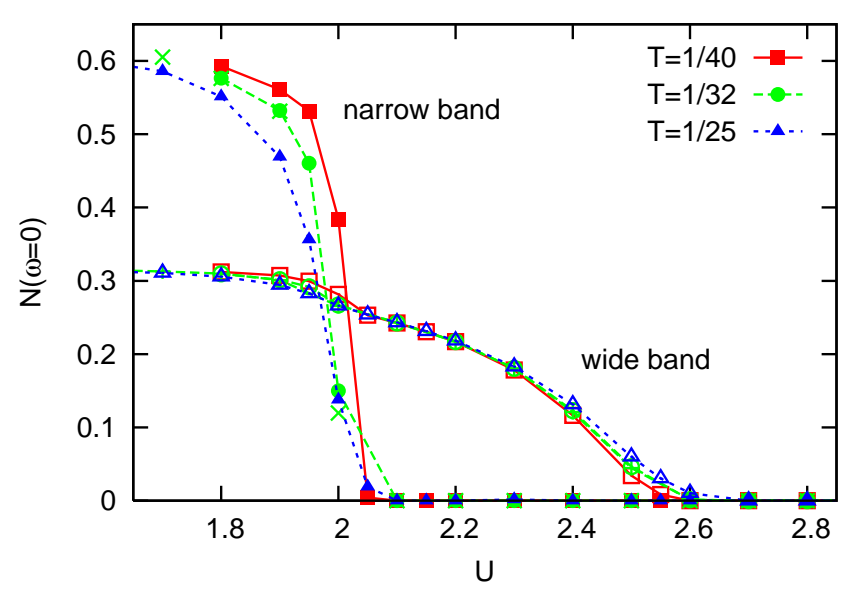

FIG. 4: (Color online) QMC estimates for value $N(0)$ of spectral function at Fermi energy versus interaction. Full/empty symbols correspond to the narrow/wide band at $\Delta \tau=0.4$; crosses to results for $\Delta \tau=0.32$ at $T=1 / 32$.

curve has a striking similarity to the narrow-band spectrum for $U=2.05$. Also noteworthy are the sharp band edges at low frequencies (where MEM is most reliable) and the rapid decay at high frequencies; apparently, the high quality of our data has kept artificial broadening to a minimum. The full dependence of $N(\omega=0)$ on interaction and temperature is better resolved in Fig. 4 which clearly exposes the orbital-selective Mott transition: at $U_{c 1}$ a sudden decay to 0 is observed only for the narrow band, while the wide-band value is reduced only by some $20 \%$. Evidently, the second band becomes insulating only for $U_{c 2} \approx 2.5$. Both transitions become sharper at lower temperatures. A discretization dependence is visible for $T=1 / 32$ only at $U=2.0$.

Finally, we discuss the intraorbital double occupancy $D_{m}=\left\langle n_{m \uparrow} n_{m \downarrow}\right\rangle$. As seen in Fig. $15 \mathrm{j}, D_{\text {wide }}$ barely shows any features near $U_{c 1}$, while $D_{\text {narrow }}$ is reduced by about $50 \%$ from $U=1.9$ to $U=2.1$. We conclude that the wide orbital remains itinerant at a point where the narrow or- 


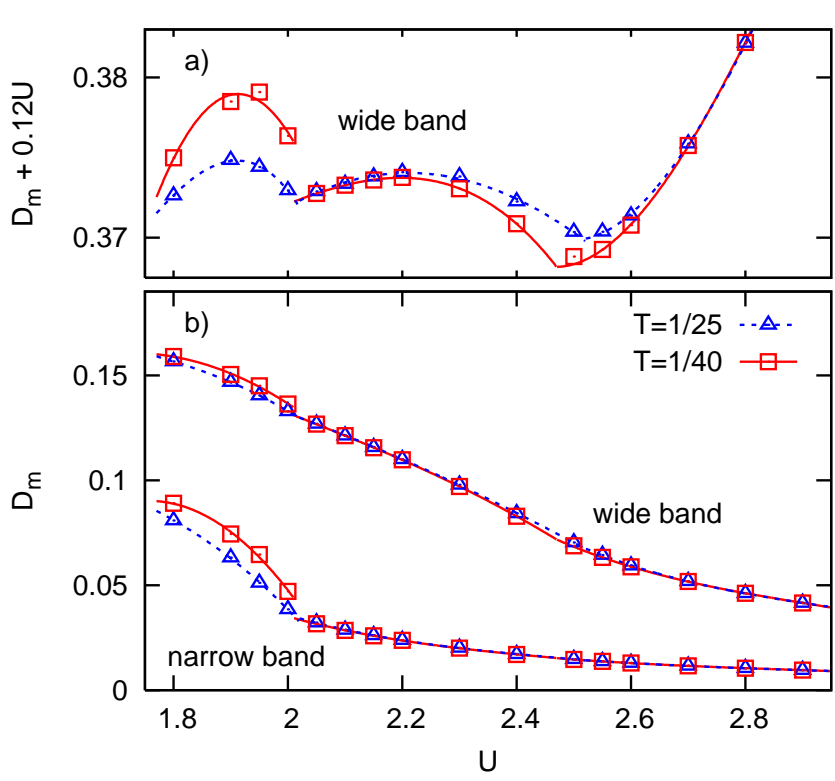

FIG. 5: (Color online) Intraorbital double occupancy $D_{m}=$ $\left\langle n_{m \uparrow} n_{m \downarrow}\right\rangle$. Lines indicate piecewise cubic fits. Upper panel: a linear offset exposes the features of the wide band.

bital is already localized. A second transition of the wide band is clearly seen only after adding a suitable linear term in Fig. 5 . Here, both transitions appear as regions of enhanced temperature dependence and as kinks. Note that up to the discontinuity at $U_{c 1}$ for $T=1 / 40$ and on a broader scale the kink is even more pronounced at $U_{c 2}$ than at $U_{c 1}$. Once again we find two phase transitions, each of which can be associated with a Mott transition of one orbital. Signatures in observables associated with the other orbital reflect the fact that phase transitions usually leave traces in every observable.
Discussion and Outlook - We considered a two-band Hubbard model with distinct band widths $W_{2}=2 W_{1}$ and interaction parameters $U, U^{\prime}=U / 2, J_{z}=U / 4$. Using high-precision QMC calculations, in which the correct high-frequency behavior of the self-energy is carefully taken into account, we showed that this $J_{z}$-model contains two successive metal-insulator transitions and can hence be considered as a minimal model for the OSMTs, observed experimentally in $\mathrm{Ca}_{2-x} \mathrm{Sr}_{x} \mathrm{RuO}_{4}$. These transitions are particularly clearly revealed by the lowfrequency behavior of the self-energy, but also visible in the spectral functions, quasiparticle weights, and intraorbital double occupancies. Our high-precision data correct earlier QMC results $9.10,11$ by Liebsch. They also contradict early ED results $\underline{14}$ by Koga et al., who may have missed the relatively narrow OSMT region due to ED's finite energy resolution. On the other hand, our results are in qualitative agreement with de' Medici et al.'s recent slave-spin mean-field phase diagram for the $J_{z}$-model, 16 which also contains an orbital-selective Mott phase, albeit at slightly larger $U$-values (at $T=0$ ).

Our results imply in particular that isotropy of the Hund exchange is not a prerequisite for the existence of OSMTs, as was suggested by Koga et al $l^{14}$ on the basis of their ED study. In future work it will be of interest to investigate the effect of other terms in the Hamiltonian, such as hybridization terms of various symmetry; first results in this direction have already been reported in Ref. 18. It would also be of interest to extend the calculations reported here to lower temperatures, to the more realistic 3-band case and to magnetic phases.

Acknowledgements - We thank K. Held, E. Jeckelmann, A. Liebsch, and K. Ueda for stimulating discussions and acknowledge support by the NIC Jülich and by the DFG (Forschergruppe 559, Bl775/1).
* Electronic address: Nils.Bluemer@uni-mainz.de

1 F. Gebhard, The Mott Metal-Insulator Transition, Springer, Berlin (1997).

2 S. Nakatsuji, Y. Maeno, Phys. Rev. Lett. 84, 2666 (2000).

3 S. Nakatsuji, Y. Maeno, Phys. Rev. B 62, 6458 (2000).

4 V.I. Anisimov, I.A. Nekrasov, D.E. Kondakov, T.M. Rice, and M. Sigrist, Eur. Phys. J. B 25, 191 (2002).

5 A. Georges, G. Kotliar, W. Krauth, and M. Rozenberg, Rev. Mod. Phys. 68, 13 (1996).

6 Z. Fang and K. Terakura, Phys. Rev. B 64, 020509(R) (2001).

7 Z. Fang, N. Nagaosa and K. Terakura, Phys. Rev. B 69, 045116 (2004).

8 M. Sigrist and M. Troyer, Eur. Phys. J. B 39, 207 (2004).

9 A. Liebsch, Europhysics Letters 63, 97 (2003).

10 A. Liebsch, Phys. Rev. Lett. 91, 226401 (2003).

11 A. Liebsch, Phys. Rev. B 70, 165103 (2004).

12 Our reanalysis of the IPT data of Ref. 11 revealed a sharp second transition at $U \approx 3.6-3.7$ for low $T \leq 0.02$.

13 A. Koga, N. Kawakami, T.M. Rice, and M. Sigrist, Phys. Rev. Lett. 92, 216402 (2004).

14 A. Koga, N. Kawakami, T.M. Rice, and M. Sigrist, cond-mat/0406457 (unpublished).

15 M. Ferrero, F. Becca, M. Fabrizio, and M. Capone, cond-mat/0503759 (unpublished).

16 L. de' Medici, A. Georges, and S. Biermann, cond-mat/0503764 (unpublished).

17 R. Arita, and K. Held, cond-mat/0504040 (unpublished).

18 A. Koga, N. Kawakami, T.M. Rice, and M. Sigrist, cond-mat/0503651 (unpublished).

19 M. Ulmke, V. Janiš, and D. Vollhardt, Phys. Rev. B 51, 10411 (1995).

20 M. Jarrell, H. Akhlaghpour, and T. Pruschke, in Quantum Monte Carlo Methods in Condensed Matter Physics, edited by M. Suzuki (World Scientific, Singapore).

21 V. Oudovenko, G. Kotliar, Phys. Rev. B 65, 75102 (2002).

${ }^{22}$ N. Blümer, Ph. D. thesis, Universität Augsburg (2002).

${ }^{23}$ C. Knecht, diploma thesis, Universität Mainz (2002).

24 M. Potthoff, T. Wegner, and W. Nolting, Phys. Rev. B 55, 16132 (1997).

25 N. Blümer and E. Kalinowski, Phys. Rev. B, cond-mat/0404568 (to be published).

26 C. Knecht, N. Blümer, and P.G.J. van Dongen (unpublished). 